Ce texte est une version préliminaire de la version publiée dans Thomas Uebel et Christoph Limbeck-Lilienau, éd., The Routledge Handbook of Logical Empiricism, Londres et New York, Routledge, Chap. 14, p. 148-156, 2022. Merci de citer uniquement la version publiée.

\title{
Conceptions of Truth in Early Logical Empiricism
}

\author{
Pierre Wagner
}

According to an old philosophical conception, truth is to be defined as an adequacy between the intellect and a thing (Thomas Aquinas) or, in a more contemporary vein, as a correspondence between language and reality. If this definition implies that truth is independent of our recognition of the correspondence, it has metaphysical implications that make it hard for an empiricist to accept. Logical empiricists have usually adhered to the view that the meaning of a sentence is or is strongly dependent on the possibility of recognizing whether this sentence is true or false. It is no wonder, therefore, that they rejected a metaphysically loaded interpretation of the correspondence theory of truth. Some of them have been tempted to reject the notion of truth altogether, to try to show that it is possible to do without it, or to adopt a definition which amounted to a conflation of truth and verification, obviously at odds with the traditional or the ordinary meaning of "truth."

No unified answer has been given to this predicament and there is no such thing as the logical empiricists' theory of truth. Although the question of the essence of truth was not a major issue in their agenda for the reason just given, truth was neither neglected nor ignored and it has even been the object of debates and disputes among members of the Vienna Circle. But they agreed that distinctions need to be made because the definition of truth depends on 
the kind of sentences (or propositions, or judgments) involved: logical, mathematical, empirical, universal, philosophical, and elementary sentences are not said to be true in the same sense and a separate discussion is required in each case. The result is a variegated set of issues, complicated by the key idea that a true sentence may have a conventional component in addition to an empirical one. This was especially important with respect to language: how is truth dependent on language or on grammar and is it possible to make the linguistic or the logical form of a sentence explicit in order to clarify what it is that makes the sentence true? Another twist in issues about truth resulted from the fact that not all grammatically correct sentences which have the form of declarative sentences were interpreted as having a truth-value. Value judgements, for example, were regarded as prescriptions or commands and philosophical statements were interpreted either as purely nonsensical, as grammatical rules or analytic sentences in disguise, as recommendations, or as mixtures of several components in need of clarification.

In their discussion of truth, logical empiricists were influenced by many authors, among whom Wittgenstein and Tarski deserve special notice. A major idea comes from Wittgenstein's Tractatus (1922): the idea that any sentence is functionally composed out of basic sentences and that the truth value of a sentence is a function of the truth values of the basic sentences out of which it is composed. Logical empiricists did not accept this idea uncritically but many of the questions about the truth of the so called "protocol sentences" were derived from it: are there elementary sentences and if so, how should they be characterized, and are they incorrigible? Wittgenstein's later idea that the truth of a sentence depends on the grammar of the language to which it belongs was also influential, as well as Wittgenstein's question of whether it is possible to describe the logical form of a sentence.

Tarski's work on the definition of a truth predicate had a no-less-important impact. In his celebrated "Wahrheitsbegriff" paper (published in Polish in 1933 and translated into 
German in 1935), Tarski presents a method for defining a predicate "true in L" for a large class of formal languages L. Four provisos are important. First, what Tarski calls a "language" is actually equipped with axioms and rules of proof so that we would rather call it a "language-system." Second, "formal" means that the rules of formation and the rules of proof only depend on the logical form of the sentences, not on their meaning (although they do have a meaning; "formal" does not mean "non-interpreted"). Third, Tarski's technique for defining truth applies to formal constructed languages, not to natural languages. Tarski argues that no adequate definition of a truth predicate is possible for natural languages because there is no way to avoid the semantic paradoxes in them. Fourth, the definition of "true in L" is formulated in an essentially richer metalanguage so that "true in L" is not definable in L itself. In what follows, we shall focus mainly on Schlick's, Carnap's, and Neurath's views on truth in the early period of logical empiricism and then discuss the immediate impact Tarski's work had on these. The evolution of their views was also deeply influenced by their mutual interactions, but unfortunately this cannot be discussed here.

\section{Schlick's conception of truth}

As early as 1910, Schlick devoted a long paper (written for his habilitation) to the question of truth, in which he defends a new and original view and rejects, among others, the idea of truth as a relation of resemblance between a representation and its object; to be true is not to be a copy of anything. The truth-bearers are not ideas but judgements, conceived as complex signs designating existing states of affairs, and a judgement is true when the designation is univocal, false if it is ambiguous. If someone says "the tree is red" while the intended tree is green, it is not known whether this judgement designates a green tree or a red tree; the judgement is not univocal, so it is false. Judgments are not isolated but connected in a system: "On one side we have a system of facts, and on the other a system of judgments. Each member of the second 
system that is coordinated one-to-one with a member of the first is said to be true." (1910/1979: 96, orig. emphasis)

In his General Theory of Knowledge (1918), Schlick again takes up the issue of truth, but this time in the context of a theory of knowledge. Judgements, still designating the existence of a relation, are also true if the designation is univocal. But Schlick now remarks that knowledge is much more than the mere truth of a judgment; knowledge presupposes the use of signs which have already been used elsewhere in the interconnected system of judgments. Not every truth is a piece of knowledge, but only those specific truths which make new connections between "old" concepts, concepts which have already been introduced by implicit definitions, i.e. by true judgments which have the conventional character of definitions. Schlick thus makes an interesting connection between truth, knowledge, and convention, now insisting that the kind of truths which have often been mistaken for synthetic a priori judgements actually have, according to him, the epistemological character of definitions.

By 1930, Schlick had fallen under the spell of Wittgenstein's strict understanding of his motto "The sense of a proposition is the method of its verification." In consequence Schlick realized that there are important sentences which do not have any recognizable truthvalue. For example, the laws of physics cannot strictly speaking be verified. As Schlick put it, borrowing Wittgenstein's terms, “at bottom a law of nature does not even have the logical character of an 'assertion', but represents, rather, a 'prescription for the making of assertions'" (1931/1979: 188). Another case of important sentences without truth values were philosophical sentences when they essentially have an elucidatory function (as in the Tractatus).

In the three lectures given under the title "Form and Content" in London in 1932 and published posthumously, the paragraphs devoted to the analysis of truth show how the 
influence of Wittgenstein's Tractatus fuses with his older views. Schlick now explicitly agrees with the traditional characterization of truth as a correspondence, but the nature of this correspondence is explained as an identity of structure between propositions and facts: "The world consists of facts, the facts have a structure, and our propositions will picture the facts correctly, they will be true, if they have the same structure." (1938/1979: 348, orig. emphasis)

Schlick mentions an objection which is sometimes raised against the conception of truth as correspondence, to the effect that a comparison of a proposition with the fact it expresses is impossible, because facts are known to us by no other means than these propositions (ibid.: 349). Schlick's answer is that propositions are given to us empirically as complex signs, which are nothing but facts in the real world, and that the comparison of two facts is something we so commonly do in our daily life that nobody could reject its possibility. Striking in this reply, as in the objection, is the apparent conflation of the two questions: "What is the nature of truth?" and "How to recognize truth?" A similar conflation seems evident here: "A proposition will be verified, its truth will be established, if the structure of the sentence is the same as the structure of the fact it tries to express" (ibid.). However, once we remember that for Schlick an unverifiable sentence is (cognitively) meaningless, the close connection he establishes between the issues of truth and verification becomes understandable.

Under the influence of Wittgenstein, Schlick offered a new defense of his view of truth and verification by the middle of the decade. Defining a proposition as a sentence "together with the logical rules belonging to them, i.e., certain prescription as to how the sentence is to be used," Schlick insisted that "in order to verify the proposition I have to ascertain whether those rules have actually been obeyed—why should that be impossible?" (1935: 67, orig. emphasis) What can be seen here is that in the mid-1930s, his view of truth as correspondence 
was still embedded in a discussion of verification and of the disputed possibility of comparing statements with facts.

\section{Carnap's treatment of truth up to and including The Logical Syntax of Language}

In his 1922 dissertation on space, Carnap followed Frege in taking truth to be one of the few basic notions which are to be regarded as undefinable. First, a judgment is defined as all that is susceptible of being true or false, and the notion of judgement is then used to define propositional functions, on which logic and mathematics are built. In The Logical Construction of the World (1928), devoted to the project of a rational reconstruction of the empirical part of science, the reconstruction of the formal part is presupposed and no specific discussion of a conception of truth is required. It must be noted, however, that here Carnap adopted an extentionalist strategy for which the notion of a truth-value is important.

In his paper "Die Antinomien und die Unvollständigkeit der Mathematik" (1934b), the English version of which was incorporated into The Logical Syntax of Language in 1937, Carnap devotes a whole paragraph to an analysis of the concepts "true" and "false". His focus is now on the careful elaboration of logical frameworks in which the reconstruction of science is supposed to take place. Because the concepts true and false "are usually regarded as the principal concepts of logic" (1934a/1937: §60b), Carnap needs to explain their place in this new project. The trouble is that the customary use of these terms allows formulations such as " $A$ is true" where " $A$ " is a sentence, which leads to contradictions related to the well-known antinomy of the liar. His first conclusion is that the predicates "true" and "false" referring to sentences of some language $L$ should be used not in $L$ itself, but in a metalanguage $M L$. The $M L$-sentence " snow is white" is true in $L$ ", where "snow is white" is a name in $M L$ of the $L$ sentence "snow is white", does not have the same paradoxical consequences. Carnap sketches the following strategy to be followed: the two predicates "true in L" and "false in L" are 
included as primitive symbols in a metalanguage $M L$ and axioms such as "each sentence of $L$ is either true or false" or "no sentence of $L$ is both true and false", which provide rules for their use, are then formulated in $M L$. (Note that today, when axioms are given for a truth predicate, they are usually formulated in $L$, not in $M L$ ).

In Logical Syntax, however, Carnap does not investigate such axiomatization of truth any further. The syntactical method which he favors excludes the use of semantic concepts such as true and false: "truth and falsehood are not proper syntactical properties" (ibid.: $\S 60 \mathrm{~b}$, orig. emphasis). Whereas "consequence," "analytic," "contradictory," "provable," and other related methodological terms are definable in a syntax-language, "true" and "false" are not. Carnap's second conclusion is that in the context of the syntactic method, the adequate strategy consists in showing how to make do without these two predicates and translations are proposed to indicate how this could be done. For example, " $A$ is true" may be translated into " $A$," and " $A$ is false" into "not $A$ " (in these cases, $M L$-sentences are translated into $L$-ones). But this deflationist technique does not cover cases such as "all theorems are true" where the number of theorem is infinite. Distinctions need to be made between different uses of "true" and "false". In logical investigations, when the truth or falsity of $A$ is determined by the axioms and the rules of the system considered, "true" may be translated as "valid," "logically valid," or "provable," and "false" as "contravalid," "contradictory," or "refutable" as the case may be, all these terms being syntactically definable (in this case, the translation takes place inside $M L$ ). Other cases are considered such as "if $A$ is true, then $B$ is true" syntactically translatable as "B is a consequence of A."

The suggested translations, however, do not cover every case. Carnap implicitly recognizes the limits of the syntactical method on this point when he writes that "the majority of ordinary sentences which make use of ['true' or 'false'] can be translated either into the object-language or into the syntax-language" (ibid., emphasis added). Whereas logical truth is 
reduced to logical validity, factual truth is not reducible to any syntactic concept and no translation is suggested for sentences such as "Everything Anna says is true."

Carnap's deflationist eliminativist attitude with respect to truth does not imply that the concepts true and false are either condemned as unclear or rejected; neither are they reduced to processes of verification or confirmation. The indicated method of axiomatization shows that truth is recognized as a legitimate concept, although no general theory of truth is formulated or even sought for in Logical Syntax or in other papers in the 1930s.

\section{Neurath's rejection of the concept of truth}

Neurath had a much less conciliatory attitude with respect to "true" and "false." Sometimes he even suggested banning these terms from the language of science or, in case we would still want to use them, to redefining them. In order to understand what motivates such a radical view, it is useful to go back some years and examine his comments on, and opposition to, the idea of truth as correspondence. Then he wrote, for example, that

statements are always compared with statements, certainly not with some "reality," nor with "things" ... If a statement is made, it is to be confronted with the totality of existing statements. If it agrees with them, it is joined to them; if it does not agree, it is called untrue and rejected. There can be no other concept of "truth" for science.

(1931a/1983: 53, orig. emphasis)

What is discussed in this quotation is the process through which statements are selected and integrated into science ("the totality of existing statements"). But the idea of a comparison with reality in the first part of the quotation clearly refers to the correspondence conception of truth and the last sentence seems to suggest — misleadingly — that what has just been said is 
meant to characterize the concept of truth. If so, some confusion would be at work here between a criterion and a definition of truth.

The same apparent confusion recurs in other papers (1931b/1983: 66; 1934/1983: 102), in which Neurath's objection to the idea of a comparison between statements and experience, or things, or reality, is formulated in a way which easily drives the reader to the conclusion that a conception of truth is defended, different from the correspondence conception and compatible with the method of a comparison between statements with statements, not with reality. As a matter of fact, this reading was by Schlick, who interpreted Neurath as defending a coherence theory of truth. Hempel did the same when he declared that "obviously, Neurath's ideas imply a coherence theory" (1935: 51). Neurath's denial and Carnap's warning against such an interpretation were of little help, probably, at least in Schlick's case, because he was convinced that the articulation of some conception of truth was needed for the defense of empiricism, unlike Neurath. In his rejoinder to Schlick, who had criticized his radical physicalism for having "no unambiguous criterion of truth" (1934/1979: 376), Neurath explained in great detail his conception of justification, which did not require any theory of truth in any way (1934/1983: 105-7). Neurath regarded the concept of truth as a remnant of metaphysics which needed to be discarded or redefined. The same was true of other “imprecise verbal clusters" (Ballungen) common in the statements of everyday language which unified science was expected to transform or reinterpret. In this context, the apparent confusion between the concept of truth and the process of selecting statements for their integration into science should be understood not as the defense of a coherence theory of truth but as reflecting Neurath's willingness to replace the concept of truth and redefine "talk of truth ... as talk of the condition of acceptability" (Uebel 2007: 233).

But what about the elementary basic statements on which other statements of science are supposed to be based? Shouldn't we regard them as indubitably true if we do not want to 
renounce the very idea of empiricism? Schlick believed that the assessment of observational reports depended on the possibility of comparing such basic statements with reality and, consequently, on a correspondence conception of truth. But to his notion of observational reports, the so called "constatations" or "affirmations" ("Konstatierungen"), as indubitable, incorrigible, first-person statements which cannot even be written down, Neurath opposed his version of third-person, revisable protocol statements, which belong to the system of science and are not supposed to depend on a specific conception of truth. To Schlick's objection that empiricism demands that observational reports should be anchored into reality through a comparison of statements with facts, Neurath replied that "reality" is just another metaphysical term which should be discarded. He insisted that the possibility of discarding protocol statements be not excluded: "We shall call a statement 'false' if we cannot establish conformity between it and the whole structure of science; we can also reject a protocol sentence unless we prefer to alter the structure of science and thus make it into a 'true' statement." (1934/1983: 102) There are no unrevisable truths for Neurath, who interpreted Schlick's quest for absolute certainty (and questions such as "Why do I accept science as true?") as metaphysical and replaced it by a conception of unified science as a social practice with a multidimensional theory of the acceptability of scientific testimony at its heart (see Uebel 2009). For Neurath, we have no reason to believe that the concept of truth is indispensable for science.

\section{Logical and mathematical truth}

In Form and Content, Schlick declared that "one has taken the most important step in philosophy if one has gained a perfect understanding of the nature of logic and its relation to reality and experience" (Schlick 1938/1979: 345, orig. emphasis). This step was taken with Wittgenstein's idea that logical propositions are tautologies, propositions that have no factual 
content and are true whatever the facts may be. To the idea that "there are no conditions for the validity of logic" (ibid.: 347, orig. emphasis). Schlick added that the same could be said of mathematics, something he had certainly not found in the Tractatus. It is not clear whether Schlick was aware of the objections which could be raised at the time against such contentions, based on Gödel's incompleteness theorem or on the plurality of existing logical systems. In 1932, Carnap was well aware of these objections and in Logical Syntax, he consequently renounced not only the program of a derivation of mathematics within elementary logic, but also the very idea of correctness in logic. Yet, he was still hoping to find an explication of analyticity which would allow interpreting mathematical and logical sentences as analytic, i.e., as logically valid and thus devoid of factual content. This did not amount to see them as true simpliciter, because no system of objects, facts, or ideal entities was recognized, with respect to which mathematical sentences could be said "true". The project was rather to show that a rational reconstruction of science is possible, in which logic and mathematics are analytic, some acceptable explication of analyticity being given: logical and mathematical sentences were not regarded as true no matter what, but as analytically true in some proposed logical framework.

An obvious objection to this program runs as follows: is it true that there is no objective realm of logical and mathematical truths or objects? And how do you know that this is the case? To see how this objection can be met, Carnap's principle of tolerance (1934a/1937: §17) — to the effect that everybody is free to build her own system of logic (including mathematics) as she wishes_-should not be read as a philosophical truth but as a decision; the decision to stop searching for the one true logic and starting investigating the "boundless ocean" of infinite possible systems (ibid.: Foreword). This first decision being taken, a second one follows: the decision to look for some specific framework in which logical and mathematical sentences can be reconstructed as analytically true sentences, for 
some proposed explication of analyticity such as the one Carnap exposes in Logical Syntax. The phrase "true by convention" has sometimes been used to characterize Carnap's conception of logical and mathematical sentences, and Carnap himself used the word "convention" (ibid.: §17). But this is misleading if it presupposes that there must be something in virtue of which these sentences should be said to be true. The radicality of Carnap's view is precisely to get rid of this very idea, logical and mathematical sentences are not true but logically valid in some language $L$.

\section{The impact of Tarski's theory of truth}

After Tarski explained to him his method for defining "true in L," Carnap quickly understood the value of his semantic approach and the use he could make of it for his own metalinguistic investigations on language-systems. For Carnap, Tarski had managed to shown that "true" was a valuable scientific concept after all, and there was no reason to try to get rid of it anymore. He encouraged Tarski to present his work at the 1935 Paris Congress on Scientific Philosophy but Tarski anticipated a negative reaction and was reluctant to do so. Carnap managed to persuade him and proposed to pave the way for Tarski's new ideas by giving a talk on truth and confirmation. In this talk, he advocated not only the distinction between true and confirmed statements but also the possibility of confronting a statement with observation (1936). A hot debate ensued among logical empiricists, between those who approved and those who rejected the use of Tarski's semantic method for defining truth. Although Neurath did not express his criticism in print, his correspondence and his interventions in private meetings, first after the 1935 Paris Congress and then again in Paris in 1937, testify of his strong disapproval (see Mormann 1999; Mancosu 2007).

Neurath had nothing to object to Tarski's logical results as such but he anticipated that the introduction of such a metaphysically loaded term as "truth" into formal studies would 
have terrible effects for empiricism: philosophers would be tempted to unduly apply Tarski's method to ordinary language and be seduced into metaphysical consequences. Tarski wrote as if he regarded the classical concept of truth to mean correspondence with reality, Kokoszyńska spoke of Tarski's method as the scientific treatment of an "absolute concept of truth," and Carnap himself justified the possibility of confronting statements with facts. All this could easily look like a vindication of Schlick's views. To Neurath's objection that Tarski's definition of truth applied only to formal languages and was of no practical interest for natural languages, Tarski replied that the same remark held for the definitions of syntactical terms given in Carnap's Logical Syntax. But Neurath's negative reactions also had deeper reasons. For him, the need to clarify concepts did not entail that unified science had to be formulated in purely formal language-systems and the quest for a rational reconstruction of science could even be interpreted as a remnant of some metaphysical ideal. Neurath's conception of empiricism was of a different kind, which took the Encyclopedia as model and required a "Universal Jargon," in which imprecise cluster terms were inevitably mixed with scientific ones. For Neurath, Tarski's definition only reflected some very specific use of the term "truth" and its use as an explication of "true" introduced more confusion than clarity.

In 1937, Neurath's suggestion was to use "accepted in the Encyclopedia" and "rejected in the Encyclopedia" instead of the mystifying "true" and "false." But he could never convince Carnap who noted that if $\mathrm{A}$ is a not-yet-decided empirical sentence, " $\mathrm{A}$ is not accepted in the Encyclopedia" is certainly not equivalent to "A is false" (Mancosu 2007). "True" and "false" as used in ordinary language were not eliminable after all and were in need of an explication, for which Tarski's definition was a useful candidate.

However, Carnap's enthusiasm for Tarski's semantics does not mean that he adopted it as it stood and simply followed his lead. Their outlooks and goals were actually very different. For one thing, whereas Tarski was interested in the methodology of the deductive 
sciences, Carnap's language-systems were aimed at the reconstruction of science, including empirical science. For another, whereas Tarski very cautiously provided an explicit definition of a satisfaction relation and a truth predicate for $L$ which presupposed no other previously accepted semantic concepts, what interested Carnap was the possibility of liberalizing metalanguages so as not to be restricted to the syntactic method anymore. For him, "true in $L$ " was hardly more than a new metalinguistic tool for the explication of methodological concepts that were discussed in the Vienna Circle, especially the distinction between formal truth and empirical truth and the concept of analyticity; by contrast, Tarski was very skeptical about the possibility of finding a definite and precise explication for these concepts. Later in the 1940s, Carnap would actually realize that the framework of Tarskian semantics was not adequate for his own projects and could not help integrate modal, inductive, and intensional logic into one single framework. He consequently turned to other semantic methods based on ideas found in Wittgenstein, Waismann, Church, and others, in which the specifically Tarskian concept of truth did not play any central role anymore. What would soon be central in this new setting (and in most of Carnap's later publications) is the notion of a state description and the idea of a sentence "holding" in a state description, not to be confused with the now common model-theoretic (and Tarskian) conception of a sentence being "true in a structure". In this non-Tarskian framework, a true state description is just one which correctly describes the real world and the focus is on L-truth, not on truth itself, a sentence being L-true if it "holds" in all state descriptions (see Wagner 2017).

\section{References}

Carnap, R. (1922) Der Raum. Ein Beitrag zur Wissenschaftslehre, Kant Studien Ergänzungshefte 56. Trans. "Space. A Contribution to the Theory of Science," in Carnap, 
Collected Works (ed. by A.W. Carus et al), Oxford: Oxford University Press, 2019, vol. 1, pp. 22-208.

--- (1928) Der logische Aufbau der Welt, Berlin: Weltkreis-Verlag. Trans. The Logical Structure of the World, Berkeley: University of California Press, 1967, repr. Chicago: Open Court.

---- (1934a) Logische Syntax der Sprache, Vienna: Springer. Rev. Ed. trans. The Logical Syntax of Language, London: Kegan, Paul, Trench Trubner \& Cie, 1937, repr. Chicago: Open Court 2002.

-- (1934b) “Die Antinomien und die Unvollständigkeit der Mathematik," Monatshefte für Mathematik und Physik, 41: 263-84.

----- (1936) "Wahrheit und Bewährung," Actes du Congrès International de Philosophie Scientifique, Sorbonne, Paris, 1935, fasc. 4, pp. 18-23.

Hempel C. G. (1935) “On the Logical Positivists’ Theory of Truth,” Analysis 2: 49-59.

Mancosu, P. (2007) "Tarski, Neurath and Kokoszynska on the Semantic Conception of Truth," in D. Patterson (ed.), New Essays on Tarski, Oxford: Oxford University Press, pp. 192-224.

Mormann, T. (1999) “Neurath's Opposition to Tarskian Semantics," in J. Wolenski and E. Köhler (eds.), Alfred Tarski and the Vienna Circle, Dordrecht: Kluwer, pp. 17-26.

Neurath, O. (1931a) "Physikalismus", Scientia 50 : 297-303. Trans. "Physicalism," in Neurath (1983), pp. 52-57.

---- (1931b) "Soziologie im Physikalismus", Erkenntnis 2: 393-431. Trans. "Sociology in the Framework of Physicalism," in Neurath (1983), pp. 58-90.

---- (1934) "Radikaler Physikalismus und 'wirkliche Welt”, Erkenntnis 4: 346-62. Trans. "Radical Physicalism and the 'Real World'," in Neurath (1983), pp. 100-114. 
----- (1983) Philosophical Papers 1913-1946 (ed. by R.S. Cohen and M. Neurath) Dordrecht: Reidel.

Schlick, M. (1910) “Das Wesen der Wahrheit nach der modernen Logik,” Vierteljahresschrift für wissenschaftliche Philosophie und Soziologie 34 : 386-477. Trans. "The Nature of Truth According to Modern Logic,” in Schlick (1979), vol. 1 pp. 41-103.

----- (1918) Allgemeine Erkenntnislehre, Berlin: Springer, 1918, 2nd rev. ed. 1925. Trans. General Theory of Knowledge, Vienna: Springer, 1974, repr. Lasalle, IL: Open Court, 1985.

(1931) “Die Kausalität in der gegenwärtigen Physik,” Die Naturwissenschaften 19: 145-

62. Trans. "Causality in Contemporary Physics," in Schlick (1979), vol. 2, pp.176-209.

----- (1934) “Über das Fundament der Erkenntnis,” Erkenntnis 4 : 79-99. Trans. “The Foundation of Knowledge,” in Schlick (1979), vol. 2, pp. 370-87.

---- (1935) “Facts and Propositions," Analysis 2: 65-70. Repr. in Schlick (1979), vol. 2, pp. $400-4$.

----- (1938) “Form and Content,”, in Schlick, Gesammelte Aufsätze 1926-1936, Vienna:

Gerold, pp. 151-250. Repr. in Schlick (1979), vol. 2, pp. 285-369.

----- (1979) Philosophical Papers (ed. by H. L. Mulder and B. van de Velde-Schlick), Dordrecht: Reidel, vol. 2.

Tarski, A. (1935) “Der Wahrheitsbegriff in den formalisierten Sprachen,” Studia Philosophica 1: 261-405, (trans. of Polish original in Trav. Varsovie 34 (1933)). Engl. trans. "The Concept of Truth in Formalized Languages", in Tarski, Logic, Semantics, Metamathematics, Oxford: Clarendon Press, 1956, pp. 152-278.

Uebel, T. (2007) Empiricism at the Crossroads: The Vienna Circle's Protocol Sentence Debate, Chicago: Open Court. 
---- (2009) “Neurath's Protocol Statements Revisited: Sketch of a Theory of Scientific

Testimony," Studies in History and Philosophy of Science 40: 4-13.

Wagner, P. (2017) “Carnapian and Tarskian Semantics,” Synthese 194: 97-119.

Wittgenstein, L. (1922) Tractatus Logico-Philosophicus (bilingual ed. Trans. by F. Ramsey and C. K. Ogden), London: Kegan Paul, Trench Trubner \& Co., 1922, rev. ed. 1933, repr. Londond: Routledge, Kegan Paul, 1961, repr. 1974. 